
\title{
A Wave Theory of the Mind: A Coherent Mind Creates a Strong Mind and Healthy Body
}

International Journal of Theoretical \& Computational Physics

Short Article

George Gerhab

Hellertown, USA
*Correspondence author

George Gerhab

Hellertown

USA

Orcid : 0000-0003-2798-4786

Submitted : 12 Aug 2021 ; Published : 25 Aug 2021

\begin{abstract}
Brain waves have been detected and measured. These waves are electro-magnetic waves that flow through the membranes of cells and nerve fibers. Amplitude and frequency modulation (AM and FM) carries the information from cell to cell while the myelin coating prevents the information from being distorted. The Nodes of Ranvier are the regeneration stations so the information wave doesn't die out. Interference of the waves creates holographic memories, coherent waves creating much stronger holograms than incoherent waves, which are far weaker than coherent waves. The autonomous nervous system carries health guided information (Placebo effect) the more coherent the signal, the stronger the health information is. Methods to create a positive, coherent mind and healthy body are given.
\end{abstract}

\section{Brain Wave Frequencies}

The following frequencies have been detected and measured in the brain. $. \gamma-35 \mathrm{~Hz}-$ concentration, problem solving . $\beta-12 \mathrm{~Hz}-35 \mathrm{~Hz}-$ speed, active, external attention, memory recall

$. \alpha-8 \mathrm{~Hz}-12 \mathrm{~Hz}$ - very relaxed, passive attention

$. \theta-4 \mathrm{~Hz}-8 \mathrm{~Hz}$ - deeply relaxed, inward focus

$. \delta-.5 \mathrm{~Hz}-4 \mathrm{~Hz}-$ sleep

Waves flow along the membranes of cells and nerve fibers The phosphorus- containing group (the polar head) of a lipid contains a dipole. When two are pointed in the opposite directions, they stick together to form a membrane.

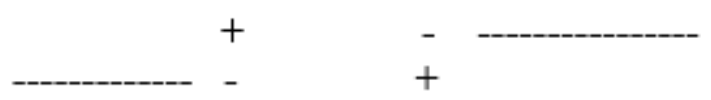

Figure 1: the + and - charges match, creating an electric bond.

These dipoles would very easily vibrate as a result of an electro-magnetic wave carrying information. The movements of the lipids is not the wave, but the medium through which the electro-magnetic wave travels through much like fiber optics. This medium slows the wave down a lot but it's still pretty fast.

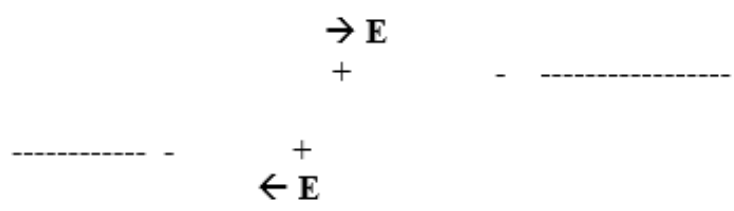

Figure 2: the electric field, E, from the electro-magnetic wave shifts the position of lipid according to the amplitude and frequency of the wave.
The amplitude and frequency of the electro-magnetic wave contains the information being sent

The amplitude modulation (AM) and frequency modulation (FM) of the electromagnetic wave contains the information that's being sent from cell to cell. The myelin coating around the nerve fibers protects this information from being distorted by the small ions (sodium, potassium, calcium, etc.) that surround the membrane.

Nodes of Ranvier are the regenerating stations for the information wave

The nodes of Ranvier contain sodium ion channels.

The information wave loses energy as it travels the dipole medium since it has to move dipoles back an forth in order to carry the information through. The nodes of Ranvier do not have a myelin coating but does contain sodium ion channels that open and close at the exact frequency and amplitude as the wave, since the channels are stimulated by the wave. The sodium ion is positively charged and the in and out motion of the sodium ion strengthens and regenerates the information wave. Without this flowing of the sodium ion, the wave would die out. (My theory of bi-polarism is that some of the sodium channels are too small to let sodium through. Lithium ion has the exact chemical properties as sodium, but it's much smaller, making up for the smallness of the channels)

Stimulated Emission of the neuron to create another wave coherent with the first

The information wave travels down to end of a dendrite where the wave excites neurotransmitters which are sent out to excite the membrane of a neuron with the exact same frequency and amplitude as the original wave. The sodium and potassium ion channels are then excited at the same frequency and amplitude. 
If the excitation is strong enough, the neuron creates a second wave in exact coherence with the original wave. In this way, the information grows instead of becoming weaker. The intensity of a coherent wave is proportional to the number of neurons involved squared, whereas the intensity of an incoherent wave (where the wave crests don't match) is proportional only to the first power of this number, $\mathbf{N}$.

Incoherent waves I : N Coherent waves I : $\mathrm{N}^{2}$

If a million neurons are involved in the transfer of the information of the wave, then the intensity of the coherent wave would be one million times the intensity of an incoherent wave with less expenditure of energy. A coherent mind is STRONG!

\section{Coherent waves create memory holograms easily}

Coherent waves are created by neurons through stimulated emission, similar to a laser (Light Amplification by Stimulated Emission Radiation) It is very easy to create a hologram with a laser (I've done it many times). You split the beam, one half goes directly to the film while the other half reflects off the object before going to the film. The two beams interfere and produce an interference pattern. When the non-information beam is shown through later, the object appears. Incoherent light, known as white light, can also make holograms, but it's much more difficult. The two beams must travel the exact same distance before a hologram can form. The distance traveled by the two coherent beams doesn't matter cause the waves are coherent everywhere not just in one place. Coherent waves can be altered by many, many kinds of distractions to produce incoherent waves.

An interference pattern consists of dark and light lines in a very complex and intricate pattern. Darks lines occur when the crests of the wave match and the light lines occur when a crest of one wave matches the trough of the other wave. Holograms contain a HUGE amount of information. Incoherent waves would be far less efficient in creating holograms than a coherent wave would. Once a hologram is formed, a coherent non-information wave would change into the memory wave by passing through the hologram.

Autonomous nervous system, the mind's connection to the body

We all know the nervous system. We use to for movement, all the senses, including pain. But, there is another nervous system, the autonomous nervous system, which acts silently. Heartbeat, liver, kidney functions, etc. are controlled by this silent partner. All cell membranes contain the same polar lipid as the nerve fiber membrane. The autonomous nervous system can transfer it wave information to the membranes of cells, and send messages to them to keep them well. If the coherent messages are distracted by many, many kinds of distractions, these messages may not be correct. If we had some control over this process, well you can imagine.
Enhancing and Directing the Placebo effect

In order to get FDA approval for a medicine, the medicine must beat the placebo, the ability of the body to heal itself. A large group of test subjects are given a sugar pill but told that it's the real medicine. The act of taking the sugar pill and believing that it's the real medicine and reenacting this procedure produces healing brain waves and more often than not the test subject gets better. In the Pfizer covid vaccine test, only 95 out of 20,000 placebo test subjects got ill when injected with the virus.

In the 1920's a French physician treated his patients with selfhypnosis and had a $98 \%$ cure rate without the use of medicine at all. I believe that he discovered a way to enhance and direct the placebo effect. I believe I can now explain his method in terms of coherent waves creating health holograms which can heal the body by sending health messages through the autonomous nervous system, not requiring medicine at all.

Self-hypnosis is not like the regular type of hypnosis which involves a hypnotist put a person under a trance, freezing the mind so that only the hypnotist's words can be heard. Self-hypnosis is a relaxation technique to calm the higher frequencies, $\gamma$ and $\beta$ which deal with the external world, so that they don't interfere with the lower frequencies becoming created by the suggestions to create coherent health holograms. No trance is involved and the conscience mind must be present all the time. The word sleep means real normal sleep, because you get closer to the $\delta$ sleep frequencies, if you get into the $\delta$ waves, you will sleep for a while. This happens when the mind is tired. To start, sit or lay in a relaxing position. Then relax each part of your body, for example, to yourself (not aloud) "legs, relax, legs relax" repeat until your legs feel relaxed, continue for the other parts of your body. Next, say to yourself, "I will now count from 1 to 10 and as I count, I will fall deeper and deeper into sleep" for example, "ONE, relax and sleep, relax and sleep, then after 5, I am in a very deep and relaxed sleep". You should now be ready for your suggestions. My first one is "My four main neurotransmitters are always balanced and coherent" this sets up any others you may have. If there's something in particular that's bother you, like cancer or any other illness or pain, zero in on it right away. The suggestion should be short, positive and goal oriented, for example "I am completely cured of illness X" do not say I'm getting cured cause this is goal oriented. I like to end my suggestions with "Every day, in every way, I'm getting better and better" this takes care of everything else. Don't overload your mind with suggestions and remember they only work within your body. The waves are way too weak to be projected outside your body, in other words, don't try to fly. If there is nothing specifically wrong with I would go with "I am in perfect health" this will maintain your health so that you don't get ill in the future.

Anything that is positive with help create a coherent mind. Healthy diet, yoga, meditation, accomplishments (doing puzzles, mowing the lawn, finishing off a work day, attending church, parties(without getting too drunk or high), cleaning the house) Whatever makes you feel good about yourself is 
a positive thing and you should make special note of it when you're done. My friend whose been doing my method for 2 years says, "the best thing about it is the self-confidence you feel that you'll never be sick again."

\section{Coherence is Power}

\section{References}

1. Marieb, Elaine, (1992) "Human Anatomy and Physiology" The Benjamin/Cummings Publishing Co, 1992.

2. Gerhab, George (2021) "Enhancing and Directing the Placebo Effect" Journal of Psychology and Neuroscience, Uniscience publishing, 2021.

3. Gerhab, George (2021) "Nikola Tesla's Theory of Frequency Ratios (369) that Produce Resonances can Explain how Self-Hypnosis can Strengthen and Direct the Placebo Effect" Journal of Psychology and Neuroscience, Uniscience Publishers.

4. Wikipedia - for brain wave frequencies. 